\title{
Understanding Inclusion within Diversity: Meaning, Model, and Practice
}

\author{
Kaja Brix ${ }^{1,2}$, Olivia Lee ${ }^{2}$ and Sorina Stalla ${ }^{2}$
}

1. National Oceanic and Atmospheric Administration. NOAA Fisheries Alaska Regional Office.

2. International Arctic Research Center. University of Alaska Fairbanks. 2160 Koyukuk Drive. Fairbanks, Alaska 99775. USA

\section{Corresponding Author:}

Kaja Brix, Ph.D.

Arctic Program Director. NOAA Fisheries Alaska Region and International Arctic Research Center University of Alaska Fairbanks. 2160 Koyukuk Dr. Fairbanks, Alaska 99775 USA

Kaja.Brix@noaa.gov

\begin{abstract}
The diversity and inclusion discussion permeates many sectors of society. Within this dialogue, science and scientists are acutely aware of the value of diversity and the need for inclusion. While demographic diversity in science has received considerable recent attention, very little research and understanding exists on inclusion. Our study presents empirical data on the meaning of inclusion using a crowdsourcing approach that sought responses to the question "What does inclusion mean to you?". The most prominent concepts were those of empathy, warmth, support, love, acceptance and curiosity; diverse perspectives; and participation. We clustered conceptual elements of inclusion into four themes: access and participation, embracing diverse perspectives, a welcome environment, and team belonging. On the basis of these data, we theorize a conceptual framework model from which inclusion may be put into practice. Our model suggests a dynamic process of inclusion operating from principal structural elements of 1 ) a foundation that involves place, access and participation, and space; 2) reciprocal engagement as an engine for inclusion; and 3) expression of inclusion as culture. The framework model demonstrates a means by which the practice of diversity can be more than shifts in demographic statistics, and instead promote the full expression of benefits derived when the many dimensions of diversity are truly included.
\end{abstract}

Keywords: diversity and inclusion, science, social pain, theory of inclusion, framework model of inclusion, practice of inclusion, meaning of inclusion 


\section{Introduction}

The study, practice, and value of diversity and inclusion figure prominently in varied realms of contemporary society. Organizations across sectors recognize the need for diversity and are taking steps toward, and considering the published recommendations on, generating greater diversity (Commission on Advancement of Women and Minorities 2001; Penaluna et al. 2017; Purrity et al. 2017; Fortune 2020). The sciences in particular are now acutely aware of the lack of diverse gender, ethnic, and race representation in scientific disciplines as has been reported by the the National Science and Technology Council (2018), the National Science Foundation (2019), and the National Science Board (2020). Science as a whole recognizes the challenges this deficit presents, not only as lack of diverse representation but also as lack of diverse contributions limiting scientific advancement (Medin and Lee 2012; Arismendi and Penaluna 2016; Catalyst 2018; Bell et al. 2019). Individual disciplines, professional scientific societies, and institutions in general are seeking to ensure science and scientists are more representative of the general population (NOAA 2015; Brix 2019; AGU 2019; DOI 2019; Dutt 2020).

A diverse workforce has better insight into the needs of the constituencies they serve, and is better able to consider multiple approaches and generate diverse ideas, solutions, and challenges. Diverse teams are more creative, innovative (Diaz-Uda et al. 2013), productive (McKinsey 2020), and operate at a higher performance level than non-diverse organizations (Reynolds and Lewis 2017). To realize these benefits, members of any group must feel that their uniqueness is valued and has a place, they must feel included (Catalyst 2014). While diversity and inclusion can appear conflated, or as diversity automatically entailing inclusion, diversity in itself does not generate inclusion (Johnson 2011; Sherbin and Rashid 2017). Inclusion is the functional partner to diversity, requiring separate attention.

Without inclusion, the sciences run a collective risk of failing to retain increasing diversity in their disciplines, labs, and communities. Instead diversity will remain a statistic, without the voices, experiences, and perspectives of the individuals who make up a diverse workforce bringing value to a particular effort. At a broader level, the scientific endeavor as a whole will suffer for lack of multiple perspectives and insights to creative efforts (Powell 2018).

The need for a concrete practice of inclusion is evidenced by insights into the impact of exclusion. Eisenberger et al. (2003) illustrated the neurological responses to exclusion through in-situ observations of study subjects' brains, as observed under functional magnetic resonance imaging, when exposed to experimental social exclusion. The study showed that the brain's neural alarm system (the anterior cingulate cortex) is the same area that activates during social exclusion as is activated during physical pain. This activation was also found to be positively correlated with self-reported social distress levels. Their study concluded that pain provoked by exclusion is a primitive indicator that something is wrong.

These neurological findings probe a deeper layer in understanding inclusion. Humans registering exclusion as pain suggests a profound impact from not being included. Under pain individuals are 
compromised in their ability to function: pain-related alterations in cognitive capacity are shown in attentional, executive, and general cognitive functioning (Moriarity et al. 2011). When exclusion occurs efforts at diversity fall short, not only of actually changing long term representation in the sciences (Puritty et. al. 2017) but in diminishing individuals' ability to perform. Practicing inclusion, therefore, may alleviate pain from exclusion and enhance individuals' ability to function, to perform at a task or a particular job, free from the limitations imposed by pain.

Exclusion is one means by which the full benefits of diverse representation are not realized. Assimilation, at the other end of this continuum, may produce the same result. Organizations seeking greater inclusion often achieve assimilation instead (Sheriden 2015). Assimilation, like marginalization, means that different voices and experiences don't count (Dutt 2020). Assimilation is the "closing of cultural and social distances" that separate one population from another and result in minimal or no difference among the participants (Alba and Nee 1997). Assimilation embodies variations of an acculturation and integration process whereby adaptation to the norms of the dominant culture is the expectation, is considered generally beneficial, and is the desired outcome for success (Gordon 1964; Portes and Zhou 1993; Xie and Greenman 2008; Gidley et al. 2010; Xie and Greenman 2011).

The process of assimilation occurs when diverse individuals are absorbed into and allowed to work within a group when they suppress their individuality and conform to the dominant culture. Inclusion, however, is the opposite; individuals are treated as insiders, welcomed and encouraged to retain their uniqueness (Sheriden 2015). It is worth noting that assimilation destroys individuality, whereas inclusion restores it (Llopis 2019). It is inclusion, not assimilation, that can therefore give power to diversity. It is inclusion that may allow us to move from "performative diversity", which focuses on quotas and statistics as a measure of diversity, to "cognitive diversity" which centers around giving space to diverse opinions and perspectives. Achievement of cognitive diversity with psychological safety for diverse perspectives is needed to leverage the value brought by diversity, push forward innovation (Post et. al. 2009), and ultimately scientific advancement.

As the understanding of diversity advances within the dialogue on diversity and inclusion (Hillgartner and Stalla 2019), our observations indicate more attention is necessary on understanding the meaning of inclusion and its practice (Brix 2019, Lee 2019). Many inclusion practices tend towards intuition rather than empirical evidence (Nair and Vohra 2015). Our study goal was to derive a practical inclusion framework by conducting empirical research to create a set of guiding principles for those seeking to generate and support an inclusive community among diverse members.

\section{Our Approach}

Critical to our design was the conscious absence of any hypothesis testing of preconceived ideas. We recognize this approach might be considered limited in some aspects; however, our approach was intentional as a means of obtaining fundamental, unfiltered data on inclusion. We 
determined that one basic question would provide an open entry point for all possibilities of fundamental understanding. This simple question was "What does inclusion mean to you?"

We presented this question on a conference sized poster with purchased access to a Shutterstock image as the backdrop (Fig. 1). The poster was displayed opportunistically as a passive data collection tool in a range of venues. We provided 3 inch $\times 3$ inch commercially available Post-it notes and writing utensils at each location to collect responses. The individual respondents were not identified or chosen. At will contributions allowed for spontaneous, voluntary, and anonymous responses. The rationale for the $3 \times 3$ inch Post-it notes was to give enough room for a response but not too much room, increasing the likelihood of elemental data.

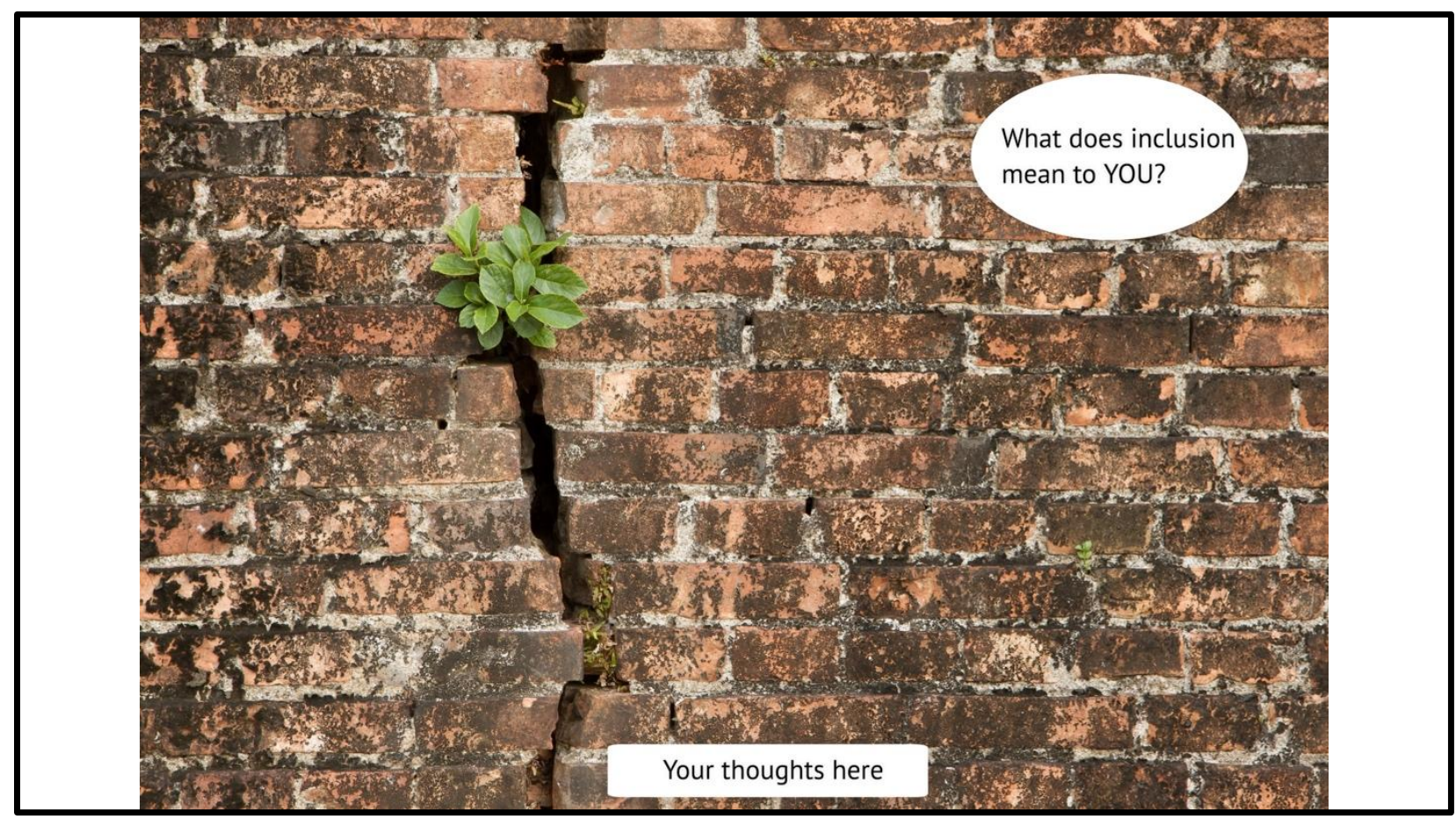

Figure 1. Inclusion poster.

The poster was placed opportunistically in 15 different venues representing a broad spectrum of society, including scientists, small business patrons, live community-based theater goers, resource agencies, education forums, an international scientific conference, local community conference, faith based conference, public community march, 55+ community center. The geographic locations were Alaska, Washington state, Arizona, up-state New York, Washington D.C., and British Columbia, Canada. Upon conclusion of the event at which the poster was placed we returned to collect all responses from each venue (Fig. 2). We pooled the total number of responses from all venues for analysis. 


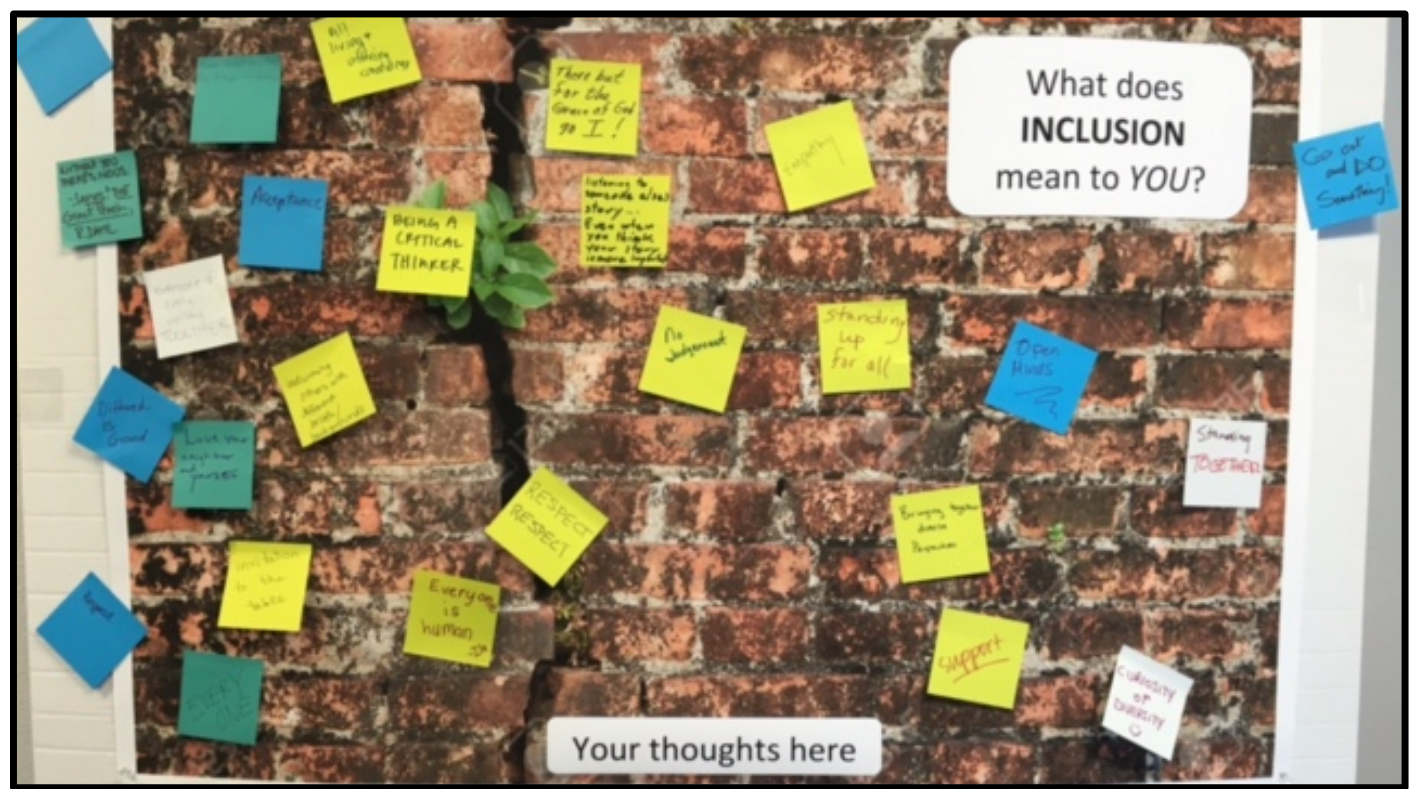

Figure 2. Poster with responses at one venue.

The data were analyzed using a social science grounded theory methodology. The grounded theory approach is a methodology for gathering, comparing, analyzing, and evaluating qualitative data (Strauss and Corbin 1994; Strauss and Corbin 1997; DePoy and Gitlin 2016). By focusing on a general topic rather than a predetermined research problem, biases arising from preconceptions are minimized. Concepts were identified in the data rather than fitting data to predetermined categories. This approach allowed for the most detailed suite of potential concepts to be revealed. No responses were discarded (although two were undecipherable). After identification of the concepts, a clustering approach of subjective evaluation of like concepts was used to group concepts and identify themes. We used the clustering approach identifying 'natural clusters', clusters exhibiting "internal cohesion and external isolation" (Milligan and Cooper 1987). We only clustered where there was clear association of words and meaning. We strove to maintain as many unique concepts as possible in an effort to elucidate the nuances and depth of meaning present in the data (Fig. 3). 


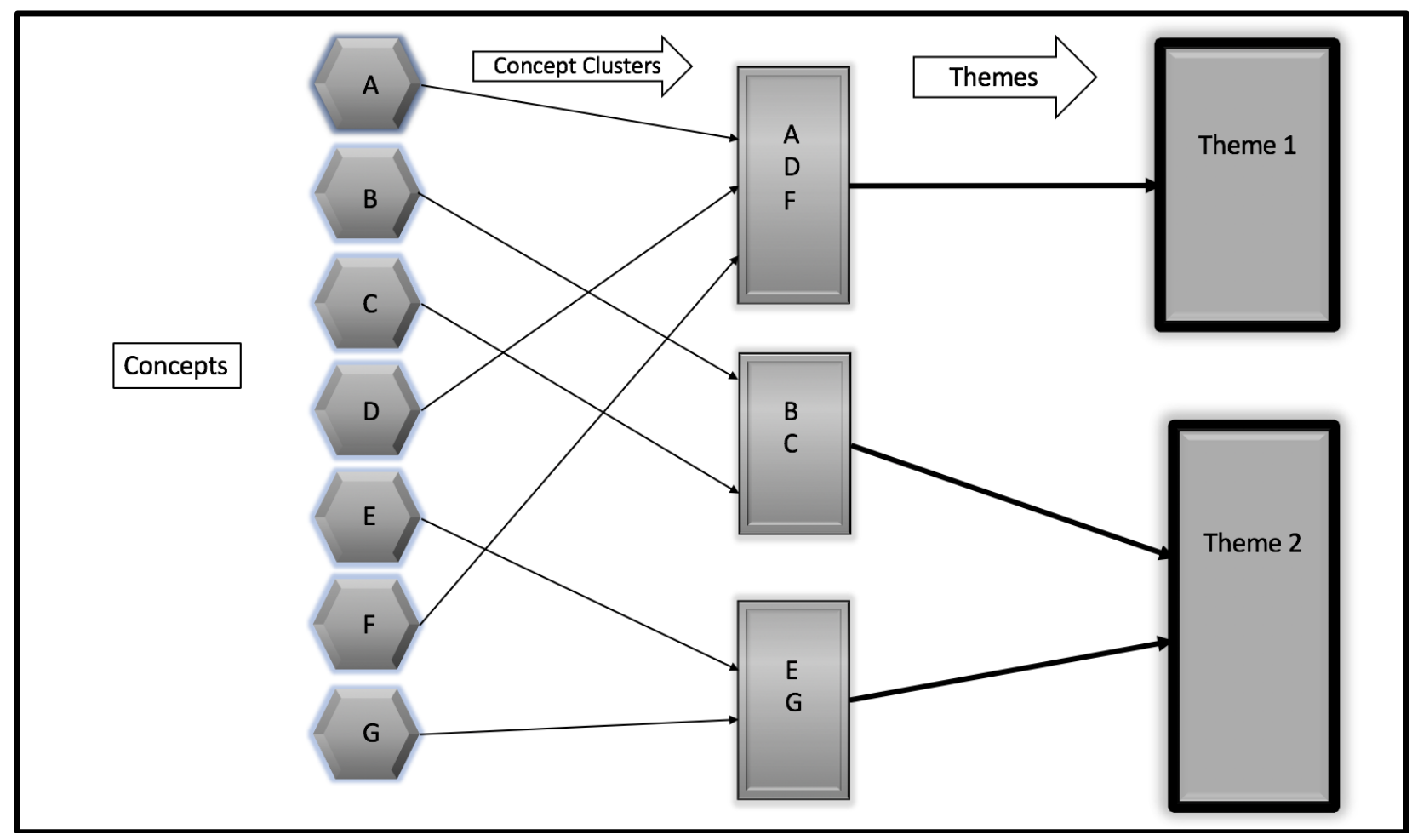

Figure 3. Schematic of concept clustering.

Each Post-it note was considered one unique response. Each Post-it response was examined individually and the concept present in the response was identified and coded with a specific word(s) identifier. For instance, the most simple level of response was one word (e.g., "respect"), and was given that concept identifier. In several cases more than one concept was present in the response. In those cases each concept within that response was identified and coded, resulting in a higher number of total concepts than unique responses.

Similarly coded concepts were grouped into a first order cluster. For example, all individual responses coded with the concept "respect" formed one first order cluster identified similarly (see Table 1). Using the first order clusters, second order clustering grouped like concepts into themes. For example, concepts coded as "participation" + "equal opportunity" + "invitation" + "seat at the table" + "asked for input" were clustered into an "Access and Participation" theme (see Table 2). The complete analysis resulted in a suite of raw data concepts, first order concept clusters, and second order themes to form a layered definition of inclusion.

\section{Empirical Data on Inclusion}

A total of 197 individual responses, with 203 concepts, were collected from the fifteen different venues. Two responses were so vague as to not allow for concept identification and were eliminated. The number of responses per venue ranged from zero to thirty-nine, with three venues receiving no responses. As the poster placement was opportunistic and the responses were at will we were not able to establish why some venues received no responses. Twenty-three first order concept clusters were identified (Table 1). 
Table 1. Concept clusters identified from the entire dataset. First order concept clusters (first column), total number of concepts per cluster (second column), and second order clusters represented as themes (third column).

\begin{tabular}{|l|c|c|}
\hline \multicolumn{1}{|c|}{ Concept Clusters } & Total & Theme Identifier \\
\hline Empathy/warmth/support/love/acceptance/curiosity & 34 & 3 \\
\hline Diverse perspectives/Open minded & 33 & 2 \\
\hline Participation & 30 & 1 \\
\hline Belonging/Team/Shared purpose & 23 & 4 \\
\hline Voice/heard & 17 & 2 \\
\hline Equal opportunity & 11 & 1 \\
\hline Invitation & 9 & 1 \\
\hline Equality & 8 & 2 \\
\hline Validate & 7 & 2 \\
\hline Respect & 6 & 3 \\
\hline Open communication & 4 & 2 \\
\hline Seat at the table & 4 & 1 \\
\hline Listening & 3 & 2 \\
\hline Open minded/have your information considered & 2 & 2 \\
\hline Asked for input & 2 & 1 \\
\hline Willingness to change & 2 & 2 \\
\hline Take Action & 2 & 2 \\
\hline Community & 1 & 4 \\
\hline Critical thinker & 1 & 2 \\
\hline Transparency & 1 & 2 \\
\hline Innovation & 1 & 2 \\
\hline Connection & 1 & 2 \\
\hline Leadership & & 2 \\
\hline
\end{tabular}

From the entire data set, the most identified concept cluster was "empathy/warmth/support/love/acceptance/curiosity" representing 34 out of the 203 individual concepts (Table 1). This concept cluster was identified with an array of words present in the individual responses. We chose a word array in this cluster to express the nuances of meaning. The depth of meaning expressed by this array is significant to understanding this cluster as a unit.

The second most identified concept cluster was "diverse perspectives/open minded" (33), with the third highest cluster being "participation" (30). Participation was clearly a singular concept in those responses with this identification. We labeled "diverse perspectives" together with "open minded" as one concept cluster to reflect the expressed nuances. These two ideas are very similar yet they also represent a duality. For instance, an individual may present a diverse perspective but it is not received unless an open mind also exists. 
The aggregation of like concepts clusters resulted in the identification of four unique themes representing the entirety of the data (Table 2). These themes are 1) Access and Participation; 2) Embrace Diverse Perspectives; 3) Welcome Environment (Culture); and 4) Team Belonging.

Table 2. Thematic representation of Concept Clusters.

\begin{tabular}{|c|l|c|}
\hline Theme Identifier & \multicolumn{1}{|c|}{ Themes } & Concepts-Total \\
\hline 1 & Access + Participation & 56 \\
\hline 2 & Embrace Diverse Perspectives & 82 \\
\hline 3 & Welcome Environment (Culture) & 41 \\
\hline 4 & Team Belonging & 24 \\
\hline
\end{tabular}

\section{What does inclusion mean?}

Our study revealed fundamental aspects on the meaning of inclusion, with a multi-dimensional characterization that has an ordered nature. The concepts grouped into themes show a clear layered and progressive aspect to inclusion. Consistent with research on social inclusion (see details in Introduction) our data show that access and participation are components of inclusion. However, our data indicate that inclusion is much more than simply granting access and participation. Our data also show that inclusion is not assimilation: the data in theme 2 on embracing diverse perspectives contradict the notion of assimilation as seen in other studies on integrating minority representation into majority settings (see Introduction). The data from our study further extend the understanding of inclusion into an expression of culture as created by a welcome environment, and the notion of a community of belonging or team.

Culture as a notion is a complicated and debated concept loosely seen as manifestations of a shared approach (Goldstein 1957). The concepts of theme 3 (Welcome Environment) as culture can be seen as an aggregation of social practices, building blocks of a society composed of collective attitudes and intentions (Balzer and Tuomela 2003). These building blocks translate into the fundamentals of a team of belonging, as captured in theme 4 .

An entire field of study on team science is providing new insight on how to build and manage diverse teams in science. A comprehensive review of the study of team science is not presented here, but other reviews from that work showed that we still have a lot to learn from how diverse teams function effectively (Hall et al. 2018). Other research that assessed feelings of trust in teams have emphasized the importance of welcoming diverse opinions (Crowston et al. 2015), but recognized that not everyone within a team will perceive benefits in the same way. For instance, graduate students in collaborative projects were found to experience less significant improvements in trust compared to principal investigators (Frescoln and Arbuckle 2015). Such findings suggest that even when participating as part of a team with a shared purpose there is value in assessing how inclusion actually functions within that team.

The concepts "community", "transparency", "critical thinker", "innovation", "connection", and "leadership" while expressed only once in the data, add an interesting dimension to our 
understanding. The concept of "critical thinker" and "innovation" suggest direction and nature of outputs that may occur as a result of an inclusive environment. The concepts of community, transparency, and connection represent functional elements as much as structural ones.

The ideas of leadership and trust, which might be intuitively expected expressions of inclusion, were notably rare (leadership appeared once) or remarkably absent (trust) in our data. In a discussion below on the role of power we suggest ideas on the role of leadership. We surmise by its absence in the data that trust may be a product of inclusion rather than part of its meaning. Trust may also be implicit in other concepts, such as belonging and community, as expressed by Team.

The interconnectedness of concepts expressed as four themes was used to create a qualitative representation of inclusion as a practice.

\section{A Conceptual Framework of Inclusion- A new perspective}

Using the data concepts and clustering in our study we theorize a conceptual model to inform the practice of inclusion (Fig. 4). The concepts and the themes comprise the principal structural elements of this model. We insert complementary functional components.

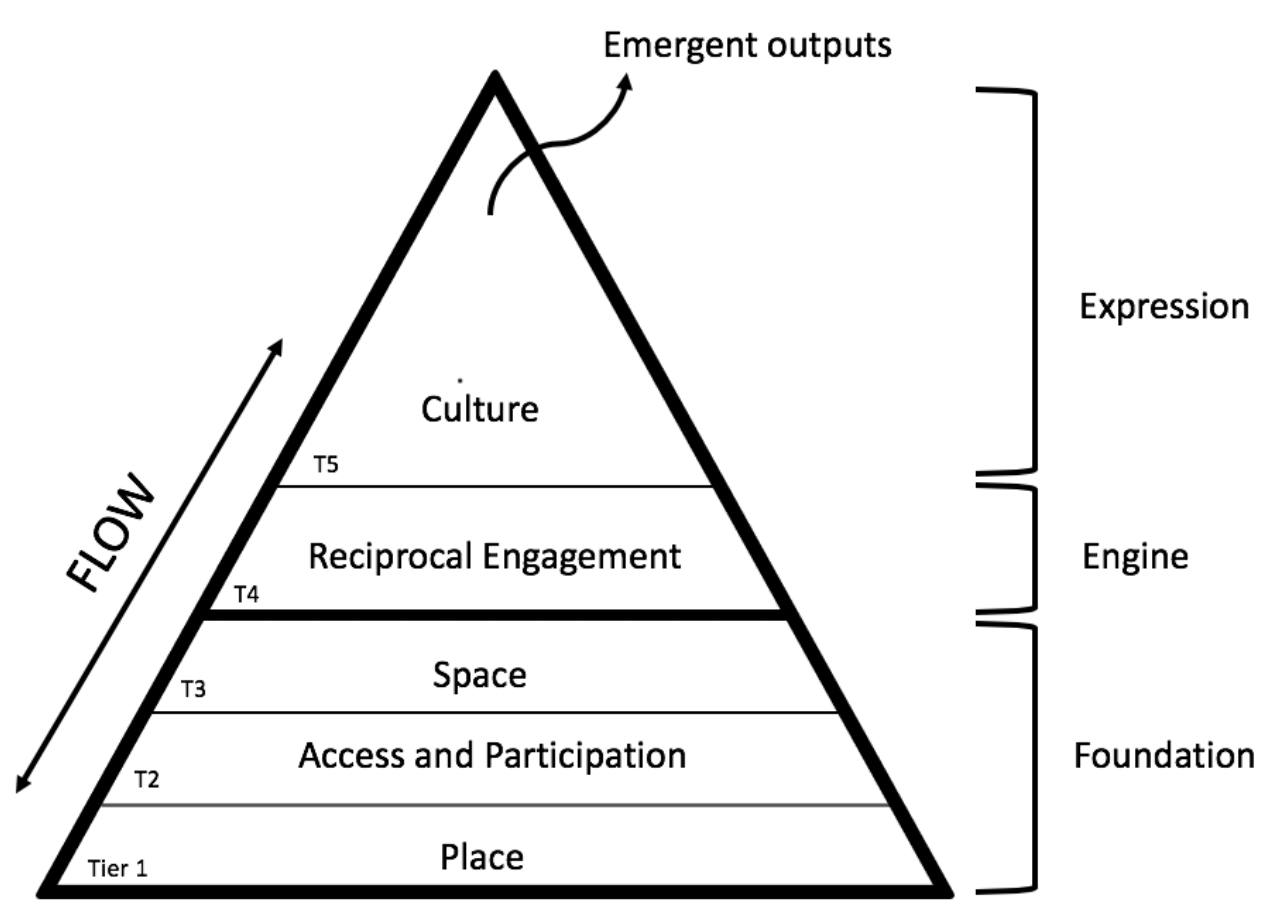

\section{Inclusion}

Figure 4. Conceptual framework of Inclusion with multi-directional flow among tiers. 


\section{Structure Function Relationships}

The principal constituent elements of inclusion are place, access and participation, space, reciprocal engagement, and culture (Tiers 1-5 respectively in Fig. 4). Place and space, together with flow and emergent outputs, are the added functional aspects fulfilling the entirety of an inclusion framework. The five tiers represent the Foundation (Tiers 1-3: place, access and participation, and space); the Engine (Tier 4, reciprocal engagement); and Expression (Tier 5 culture). Flow (see Fig. 4) is the mechanical aspect of inclusion that allows the principal constituent elements to work as a whole. The entirety of inclusion generates emergent outputs when individuals function in inclusion. We further describe these elements and the functional relationships.

The model assumes a hierarchy of tiers in which the prior tiers are necessary for the subsequent tiers. However, as represented by 'flow' (discussed below) there is fluidity within the structure and its function.

\section{Place}

Place (Tier 1 ) is the basic requirement of inclusion. A 'place' provides the necessary vessel in which inclusion occurs. The existence of a place for interaction among individuals is considered as a physical location, or in current times, a virtual meeting place. Characteristics of a place (e.g. size, decor) play a role in the atmosphere for social interaction and learning (Todino et al. 2016). This is an important consideration in identifying places specifically to encourage an inclusive culture. Without 'place' there is merely a random dispersion of individuals. Lack of attention on place precludes execution of the subsequent tiers of inclusion.

\section{Access and Participation}

Access and participation in Tier 2 is derived directly from our significant concept cluster and Theme 1 in the data. The significance of access and participation, as discussed in earlier sections of this paper, is supported by existing literature on social inclusion of minority representation into majority settings. However, as Nakata (2012) discusses, widening access and participation does not in itself lead to successes in social inclusion. Inclusion fundamentally assumes the need for access and participation but more elements are necessary for minority success in majority settings (Nakata 2012).

\section{Space}

The functional element we label 'space' is the final tier in the foundation. Space might commonly be thought of as concrete e.g. a classroom, or a conference; however, our conceptualization of space is a fluid one. Space in this meaning deserves more illustration to explain our idea of its role in inclusion. Space is the intangible aspect that exists between two people or a group of people in a place. Space allows the production of certain characteristics that we see in our data: it is the element that allows exchange of diverse perspectives to occur. It is the realm that we all intuitively recognize when our ideas are supported, voices are heard, and listening happens, where there is open-mindedness, validation, and where a willingness to change occurs (Theme 2 data concepts). 
Space is actor dependent: it is the 'place in between' where something happens. It is generated by behavior, intuitively sensed when it exists, and unconsciously noticed when it is absent. In this sense, space is created through the practice of concepts expressed in Welcome Environment (Theme 3).

Space, unbounded and multi-dimensional, allows for possibility. It allows movement of concrete elements i.e. ideas and concepts, and facilitates elements seen in Themes 2-4: transparency, critical thinking, community, belonging, and innovation. When space between does not exist we close down the possibility of open minded embrace of others' perspectives even while access and participation are present. We suggest that without space, the tendency towards assimilation becomes the default operational mode, effectively precluding the full dimensions of inclusion. Space between resists assimilation by holding difference in dynamic tension.

Our temptation with space is to fill it. Closure of space reduces the scope of possibility by collapsing differences. Instead, in resisting this closure we create greater latitude for unknown possibilities to emerge. Space is necessary to both preserve and also to connect differences. By making room for difference we allow for reciprocal engagement (Tier 4, Theme 2). In this view we also need the full effect of time for space to perform its function. The press of time may well be the enemy of space in our notion of inclusion. Harvey (1989, in Urry 2001), in developing ideas on a "time space compression", drew on Marx's thesis of the "annihilation of space by time". If this compression exists, full attention is necessary to allow time its role in generating space and enabling its function.

\section{Reciprocal Engagement and Culture}

Reciprocity as a form of engagement, derived from Theme 2 concepts, is the engine of this model. Reciprocity is "the giving of benefits to another in return for benefits received" (Molm et al. 2007). Reciprocal engagement is the functional aspect of the concepts illustrated by Theme 2 in our data: voice and listen, of giving with the expectation that you will also receive, trusting that when you speak you will truly be heard. Reciprocal engagement must be practiced and sustained. Amado et al. (2013) observed in disability inclusion that "regular contact in integrated environments, with opportunities for meaningful interaction" was the overriding factor in increasing social inclusion.

Various expressions of reciprocal engagement feature strongly in our data. A specific expression from the data -- "know my name" -- demonstrates not just a superficiality but the depth of engagement in a reciprocal form. Humans seek to be known in all fragments of their identity (Fiske 2019). When we seek to know someone's name there is a representation of depth, one in which we also give permission to be known in our own individuality. Making those narratives available to and received by others in their full complexity is the inclusive exercise of diversity (Fiske 2019).

Falk and Fischbacher (2001) model reciprocal action as the behavioral response to an originating action that is perceived as either kind or unkind. They note that the more an action is considered 
kind or unkind the more it will be rewarded or punished, respectively. The action is evaluated based on two factors: 1) the outcome, and 2) the motivation (Falk and Fischbacher 2001). These two factors suggest the recognition of an inherent self and other that are in constant dynamic engagement. Unlike a dictator game where the power rests unilaterally and each actor has a defined role, in a reciprocal arena each actor inhabits both the role of initiator and that of receiver. According to Molm (2010), all actors in a reciprocal network can initiate exchange with another actor at any given time: "some initiations may be reciprocated immediately, others later, and some never." The dynamic nature of non-transactional reciprocity builds social capital and network ties that evolve over time and are not bounded by negotiated or transactional strictures (Molm 2010).

Reciprocal exchange demonstrates the profound impact on social relationships, "not only for exchange and power but for the emergence of trust and solidarity" (Molm 2010). The result within a group is stronger integrative bonds of trust, affective regard, and solidarity (Molm 2010; Molm et al. 2007).

The social bonds of solidarity represent the expression of an inclusive culture as demonstrated by elements in themes 3 and 4 of our data and presented in Tier 5 of the model. Culture in our model is the full expression of inclusion. As identified by Baldwin et al. (2006), culture is a complex notion of systematic learned and patterned nature. This suggests reinforcement of behavior among the members. This reinforcement leads us in this model to identify feedback loops occurring between and among the tiers. Inclusion as a process means there is continual creation and re-creation in a dynamic form. This nature also likely yields a transformative capacity of inclusion, situated in this study as concepts of innovation, critical thinking, and transparency.

Flow

Taking a holistic view of this model and our data we assert that inclusion in its entirety is fundamentally dynamic. This assertion diverges from the concepts of social inclusion seen in the literature (see prior references). This dynamic is represented by a quality we label 'flow'.

Flow captures two ideas. The first is the idea of movement between and among the tiers. The model in practice is not a stepping stone of hierarchy. Rather, each tier is dependent on the others and in turn influences the others, bi-directionally. Feedback between and among the layers must exist for the whole to function. In practice we must ensure that each tier exists and is in full function. This means, for example, even in reciprocal engagement (T4) or in the expression of culture (T5) we must be certain to address and re-address the full presence and execution of preceding tiers. The second idea of flow is as a state of mind, characterized by focus, absorption, and full engagement which allows unsurpassed performance (Csikszentmihalyi 1990). Flow, both as a holistic element of the model and as a feature of individual engagement, is necessary in the full expression of inclusion.

\section{Emergent outputs}

Finally, this full expression of inclusion enables the last component represented in our framework model: emergent outputs. We posit that emergent outputs, as innovative and novel products, 
are made possible when diverse membership operates according to the tiers, in an inclusive manner. Inclusion can valuably exist as an end in and of itself to the unique individual and collective benefit of the participants. Inclusion can also have a purpose for the kinds of endeavors generated by science. Science, the discovery of something new, depends on such emergence. Arguably, the best of this scientific innovation are emergent ideas, those not conceived by any one individual but rather by a whole that is greater than the sum of its parts (Holland 1998).

\section{The role of power}

While not explicitly recognized as a structural component per se of our inclusion model, power is an embedded feature in each layer, worthy of some attention. The manifestation of power is represented in different ways in different tiers. On the foundational level, power exists in a traditional hierarchical representation. In a segmented understanding of inclusion whereby a minority representation is granted access and participation by a majority representation, power rests unilaterally with the majority. Power may be implied or overt. Power does not change hands even through participation of newcomers, perhaps until those newcomers are embedded in the group, only to be exercised in a consistent form for other newcomers.

We suggest that the reciprocal engagement of our inclusion model enables a shift in the perception and exercise of power away from a hierarchical and uni-directional application to a dispersed form. An important consequence of reciprocal exchange is that it reduces the differences in how actors in an unequal power relation perceive each other (Molm 2010). Reciprocal exchange enables actors to overcome the divisions created by power through a diminished use of power, as trust and affective bonds that promote productive exchange relations are developed (Molm et al. 1999). The effect of inclusion may therefore result in a greater feeling of equality among those of unequal power, not because of the elimination of power but because of its diminished use. Power in full inclusion may become the power of the whole rather than of the few.

Traditional power dynamics in science have had significant implications for creating environments that are far from inclusive, and that sometimes propagate dangerous situations where harassment occurs (NASEM 2018). One of the key needs to improve equity in science involves restructuring institutional power dynamics to place, for example, more emphasis on supporting victims of harassment, rather than avoiding institutional liability (Herbers et al. 2019), and rethinking hierarchical power structures (NASEM 2018). Our model suggests one means by which power may be rearranged in truly inclusive environments.

\section{Practicing inclusion}

Resistance to change is difficult to overcome, and not all organizations may be ready to break existing cultural norms in order to rebuild in a way that supports an authentically inclusive culture. Some organizations may come to the conclusion that they do not yet have that capacity to embrace an anti-fragile approach (Taleb 2012), where there may be uncertainty and chaos in adopting a new culture, a culture that holds transformative potential. Organization leaders may realize that they fall closer to the 'robust' (Taleb 2012) response to cultural change, in that disruption to business-as-usual is primarily deflected in order to retain the same existing core 
values or culture. Assessment of organizations' state of readiness to implement an inclusive culture is important, and may include developing a realistic timeframe to reach inclusion goals, budgeting for resources to build capacity (e.g. personnel training, creating and executing inclusive places), and conscious attention on inclusion as a practice.

\section{Conclusion}

The emergent themes of this research and their formation into a framework demonstrate the dynamic nature of inclusion. The tiers of the framework are not achieved in isolation nor are they isolated goals. They illustrate that inclusion is not a static marker of a destination but touchstones of a fluid journey. They also show that while inclusion is the functional partner to diversity, achieving inclusion is a distinct process that requires us to look beyond traditional diversity metrics and into the multiple spaces where inclusion exists and can be built: inside people (perception), between people (relational and behavioral), and outside people (workplace and organization) (Young 2019). As exemplified by our model, it also requires a process with both necessary and sequential steps.

Leadership can play a key role in advancing change and opening up a space for inclusion. Power, when used to create space, can be a transitional tool and a means to create a cultural shift. This may take the form of a power holder actively creating access, pushing for participation, and opening up- and holding- a space for engagement. Reciprocal engagement can grow from this space and create a shift to a culture that does not require a leader or power holder to actively generate space, but rather a culture where that space is a central and defining feature. The role of power in creating a transitional shift is most important in places resistant to this change.

An inclusive culture cannot be built on top of an old culture characterized by exclusion. It must emerge from a strong foundation that includes "unlearning" and "undoing" as much as it requires "doing" (Interaction Institute for Social Change 2020). Inclusion, built intentionally with a particular focus on reciprocal engagement, has the power to transform and create deep and lasting change. It has the power to transform us as individuals when we open ourselves up to other ideas and ways of thinking and to transform the institutions we are a part of. The individual agency that each of us has to cultivate inclusion and build inclusive communities is also the power that draws out the benefits of diversity. Actualizing these benefits requires flipping the paradigm that diversity precedes or leads to inclusion, and recognizing that inclusion is at the center of building diversity. It is inclusion within diversity that yields innovation and discovery. 


\section{References}

Alba R., \& Nee, V. (1997). Rethinking assimilation theory for a new era of immigration. International Migration Review. 31(4): 826-74.

Amado, A. N., Stancliffe, R. J., McCarron, M. \& P. McCallion. (2013). Social Inclusion and Community Participation of Individuals with Intellectual/Developmental Disabilities. Intellectual and Developmental Disabilities, Vol. 51, No. 5, 360-375.

American Geophysical Union. (2019). AGU's Ethics, Diversity and Inclusion Program.

Arismendi, I., \& Penaluna, B.E. (2016). Examining diversity inequities in fisheries science: a call to action. BioScience, 66:584-591.

Baldwin, J. R., Faulkner, S. L., \& Hecht, M. L. (2006). A moving target: The illusive definition of culture. In Redefining Culture (pp. 27-50). Routledge.

Balzer, W., \& Tuomela, R. (2003). Collective intentions and the maintenance of social practices. Autonomous Agents and Multi-Agent Systems, 6(1), 7-33.

Bell, A. M., and Chetty, R., and Jaravel, X., and Petkova, N., \& Van Reenen, J. (2019). "Who Becomes an Inventor in America? The Importance of Exposure to Innovation," The Quarterly Journal of Economics, vol 134(2), pp 647-713.

Brix, K., 2019. Introduction to the Special Issue on Diversity and Inclusion in Fisheries. Fisheries. Vol. 44. No. 8. 351-352.

Catalyst. 2014. Report: Inclusive Leadership: The View From Six Countries. (May 7, 2014.)

Catalyst. 2018. Quick Take: Why Diversity and Inclusion Matter (August 1, 2018).

Csikszentmihalyi, M. (1990). Flow: the Psychology of Optimal Experience. CreateSpace Independent Publishing Platform. 456 pp.

Commission on the Advancement of Women and Minorities in Science, Engineering, and Technology Development. (2001). Land of Plenty: Diversity as America's Competitive Edge In Science, Engineering, And Technology. Leadership and Management in Engineering, 1(4), 27-30.

Crowston, K., Specht, A., Hoover, C., Chudoba, K. M., \& Watson-Manheim, M. B. (2015). Perceived discontinuities and continuities in transdisciplinary scientific working groups. The Science of the Total Environment, 534: $159-172$. 
Department of Interior. US Department of the Interior Diversity and Inclusion Strategic Plan. (2019).

DePoy, E. \& Gitlin, L. (2016). Introduction to Research: Understanding and Applying Multiple Strategies. (Fifth Edition).

Diaz-Uda, M., Medina, C., \& Schill, B. (2013). Diversity's New Frontier. Deloitte Insights. Deloitte.com.

Dutt, K. (2020). Race and racism in the Geosciences. Nature Geoscience, 13(1), 2-3.

Eisenberger, N. I., Lieberman, M. D., \& K. D. Williams. (2003). Does Rejection Hurt? An fMRI Study of Social Exclusion. Science. 302 (5643), 290-292.

Falk, A. \& Fischbacher, U. (2001). A Theory of Reciprocity. CEPR Discussion Paper No. 3014.

Fiske, S. (2019). "Where are you really from?" and other invasions of narrative space. Fisheries. Vol. 44. No. 8. 382-383.

Fortune. 2020. The 100 Best workplaces for diversity. Fortune.com.

Frescoln, L. M., \& Arbuckle, J. G., Jr. (2015). Changes in perceptions of transdisciplinary science over time. Futures, 73: 136-150.

Gidley, J., Hampson, G., \& Wheeler, L. (2010). From Access to Success: An Integrated Approach to Quality Higher Education Informed by Social Inclusion Theory and Practice. Higher Education Policy, 23, 123-147.

Goldstein, L. J. (1957). On defining culture. American Anthropologist, 59(6), 1075-1081.

Gordon, M. (1964). Assimilation in American Life. New York: Oxford University Press.

Hall, K. L., Vogel, A. L., Huang, G. C., Serrano, K. J., Rice, E. L., Tsakraklides, S. P., \& Fiore, S. M. (2018). The science of team science: A review of the empirical evidence and research gaps on collaboration in science. American Psychologist, 73(4), 532.

Harvey, D. (1989). The Condition of Postmodernity. Oxford: Blackwell as cited in Urry, J. (2001). The sociology of space and place. The Blackwell companion to sociology, 3-15.

Herbers, J.M, Metcalf, H.E., \& Williams, R.L. (2019). Identity-based Harassment: a workshop on emerging research themes. ADVANCE Resource and Coordination Network, Washington, DC.

Hillgartner, M., \& Stalla, S. (2019). Status of Diversity in the Fisheries Profession. Fisheries. Vol. 44. No. 8. 349-388. 
Holland, J. H. (1998). Emergence: From Chaos to Order. Addison-Wesley, Redwood City, CA Interaction Institute for Social Change (2020). Power Equity and Inclusion. Interactioninstitute.org

Johnson, D. R. (2011). Women of color in science, technology, engineering, and mathematics (STEM). New Directions for Institutional Research, (152), 75-85.

Lee, O. (2019). What does diversity require of us. Fisheries. Vol. 44. No. 8. 375.

Llopis, G. (2019). Assimilation Destroys Individuality, Inclusion Restores It. Forbes.

McKinsey. (2020). Diversity Wins: How Inclusion Matters. May 2020. Mckinsey.com

Medin, D. L., \& Lee, C. D. (2012). Diversity Makes Better Science. Association for Psychological Science. May/June 2012.

Milligan, G. W., \& Cooper, M. C. (1987). Methodology review: Clustering methods. Applied psychological measurement, 11(4), 329-354.

Molm, L. D. (2010). The structure of reciprocity. Social psychology quarterly, 73(2), 119-131.

Molm, L. D., Peterson, G., \& Takahashi, N. (1999). Power in negotiated and reciprocal exchange. American sociological review, 876-890.

Molm, L. D., Schaefer, D.R., \& Collett, J.L., (2007). "The Value of Reciprocity." Social Psychology Quarterly 70: 199-217

Moriarity, O., McGuire, B.E., \& Finn, D.P., (2011). Progress in Neurobiology. 93385-404

Nair, N. \& Vohra, N. (2015). Diversity and Inclusion at the Workplace: A Review of Research and Perspectives. Indian Institute of Management, No.2015-03-34

Nakata, M. (2012). CDSI Plenary: Widening participation, social inclusion, closing the gap. Journal of Academic Language \& Learning. Vol. 6, No. 12, 1-18.

National Oceanic and Atmospheric Administration. Education Strategic Plan 2015 - 2035. (2015).

National Academies of Sciences, Engineering, and Medicine. (2018). Sexual harassment of women: climate, culture, and consequences in academic sciences, engineering, and medicine. National Academies Press. 
National Science Board. (2020). Science and Engineering Indicators. The State of US Science and Engineering.

National Science Foundation, National Center for Science and Engineering Statistics. (2019). Women, Minorities, and Persons with Disabilities in Science and Engineering: 2019. Special Report NSF 19-304. Alexandria, VA.

National Science and Technology Council. (2018). Charting a Course for Success: America's Strategy for STEM Education. A Report by the Committee on STEM Education of the National Science \& Technology Council.

Penaluna, B. E., Arismendi, I., Moffitt, C. M., \& Penney, Z. L. (2017). Nine proposed action areas to enhance diversity and inclusion in the American Fisheries Society. Fisheries, 42:399-402

Portes, A., \& Zhou, M. (1993). The New Second Generation: Segmented Assimilation and Its Variants. The ANNALS of the American Academy of Political and Social Sciences, 1993;530:74-96.

Post, C., De Lia, E., DiTomaso, N., Tirpak, T. M., \& Borwankar, R. (2009). Capitalizing on thought diversity for innovation. Research-Technology Management, 52(6), 14-25.

Powell, K. (2018). Science benefits from diversity. Nature. 558, 19-22.

Puritty, C., Strickland, L.R., Alia, E., Blonder, B., Klein, E., Kohl, M.T., McGee, E., Quintana, M., Ridley, R.E., Tellman, B. \& Gerber, L.R., (2017). Without inclusion, diversity initiatives may not be enough. Science, 357(6356), pp.1101-1102.

Reynolds, A., \& D. Lewis. 2017. Teams solve problems faster when they're more cognitively diverse. Harvard Business Review. hbr.org. March 2017.

Sherbin, L., \& Rashid, R. (2017). Diversity Doesn't Stick Without Inclusion. Harvard Business Review, 1.

Sheridan, B. (2015). Diversity isn't our ultimate goal. Inclusion Is. Business Learning Institute. August 2015.

Strauss, A., \& Corbin, J. (1994). Grounded theory methodology. Handbook of qualitative research, $17,273-85$.

Strauss, A., \& Corbin, J. M. (1997). Grounded theory in practice. Sage.

Taleb, N. (2012). Antifragile: Things that gain from disorder. New York. Random House.

Todino, M. D., Aiello, P., \& Sibilio, M. (2016). Flexible classrooms for inclusive education. In ICERI2016 Proceedings 9th International Conference of Education, Research and Innovation Dates (pp. 1674-1678). 
Urry, J. (2001). The sociology of space and place. The Blackwell companion to sociology, 3-15.

Xie, Y., \& Greenman, E. (2008). Is Assimilation Theory Dead? The effect of assimilation on adolescent well-being. Social Science Research, 37(1): 109-137.

Xie, Y., \& Greenman, E. (2011). The Social Context of Assimilation: Testing implications of segmented assimilation theory. Social Science Research. 40(3): 965-984.

Young, M. (2019). More Than A Feeling: Measuring Workplace Inclusion. Work Design Magazine. Workdesign.com 